\title{
openheart Osteopontin predicts clinical outcome in patients after treatment of severe aortic stenosis with transcatheter aortic valve implantation (TAVI)
}

\author{
Matthias Lutz, ${ }^{1,2}$ Nora von Ingersleben, ${ }^{1}$ Moritz Lambers, ${ }^{1}$ Mark Rosenberg, ${ }^{1,2}$ \\ Sandra Freitag-Wolf, ${ }^{3}$ Astrid Dempfle, ${ }^{3}$ Georg Lutter, ${ }^{2,4}$ Johanne Frank, ${ }^{1}$ \\ Peter Bramlage, ${ }^{5}$ Norbert Frey, ${ }^{1,2}$ Derk Frank ${ }^{1,2}$
}

\section{To cite:}

Lutz M, von Ingersleben N, Lambers M, et al. Osteopontin predicts clinical outcome in patients after treatment of severe aortic stenosis with transcatheter aortic valve implantation (TAVI). Open Heart 2017;4:e000633. doi:10.1136/ openhrt-2017-000633

Received 28 March 2017 Revised 12 April 2017 Accepted 25 April 2017

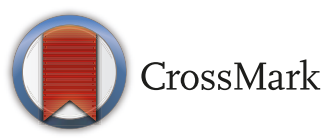

${ }^{1}$ Department of Internal Medicine III (Cardiology and Angiology), University Hospital Schleswig-Holstein, Kiel, Schleswig-Holstein, Germany ${ }^{2}$ German Centre for Cardiovascular Research, DZHK Partner Site Hamburg/Kiel/ Lübeck, Kiel, Germany ${ }^{3}$ Institute of Medical Informatics and Statistics, Christian-

Albrechts University of Kiel, Kiel, Germany

${ }^{4}$ Department for Cardiovascular Surgery, University Hospital Schleswig-Holstein, Kiel, Germany

${ }^{5}$ Institute for Pharmacology and Preventive Medicine, Cloppenburg, Germany

Correspondence to Dr Derk Frank, Department of Cardiology and Angiology, University Hospital SchleswigHolstein, Campus Kiel ArnoldHeller-Str. 3, Haus 6, 24105 Kiel, Germany; derk.frank@uksh.de

\section{ABSTRACT}

Objective 0steopontin (OPN) is an extracellular matrix protein that plays an integral role in myocardial remodelling and has previously been shown to be a valuable biomarker in cardiovascular disease. Because of the concentric myocardial hypertrophy associated with severe, symptomatic aortic stenosis (AS), we hypothesised that OPN expression may have a prognostic value in patients undergoing transcatheter aortic valve implantation (TAVI).

Methods We prospectively included 217 patients undergoing TAVI between February 2011 and December 2013 with a median follow-up of 349 days. Twenty healthy individuals from the same age range free from structural heart disease served as controls. The primary endpoint for the analysis was survival time.

Results Median preprocedural OPN levels $(675 \mathrm{ng} / \mathrm{mL}$; IQR 488.5-990.5 $\mathrm{ng} / \mathrm{mL}$ ) were significantly higher in patients with severe aortic valve stenosis compared with healthy controls $(386 \mathrm{ng} / \mathrm{mL}$; IQR $324.5-458, \mathrm{p}<0.001)$. Patients with increased OPN values showed at baseline a decreased 6 min walk test performance, increased rates of atrial arrhythmia, and an increased risk of death during follow-up (HR 2.2; $95 \% \mathrm{Cl} 1.3$ to 3.5 for the comparison of the highest vs lowest OPN quartile). Multiple Cox regression analysis demonstrated that OPN improves the prediction of an adverse prognosis further than $\mathrm{N}$-terminal probrain natriuretic peptide.

Conclusions OPN levels at baseline are associated with adverse outcomes in patients with severe, symptomatic AS undergoing TAVI.

\section{INTRODUCTION}

Transcatheter aortic valve implantation (TAVI) has become a well-established method for the treatment of severe symptomatic aortic stenosis (AS) in inoperable and high-risk patients. ${ }^{1-3}$ This assessment is based on risk stratification for which the logistic EuroSCORE (Log ES) and the Society of Thoracic Surgeons (STS) PROM Score are used $^{4-7}$ in addition to further patient-related parameters such as aortic

\section{KEY QUESTIONS}

What is already known about this subject?

- Preprocedural risk stratification of patients with symptomatic aortic stenosis undergoing transcatheter aortic valve implantation (TAVI) remains difficult. There is an ongoing controversy whether biomarkers might add relevant information.

What does this study add?

- Preprocedurally elevated osteopontin levels are significantly associated with adverse outcome after TAVI. Thereby osteopontin appears to be superior to the established biomarker $\mathrm{N}$-terminal probrain natriuretic peptide.

How might this impact on clinical practice?

- This study provides further evidence that novel biomarkers such as osteopontin might improve preprocedural risk stratification in patients undergoing TAVI. Thus, elevated osteopontin levels might trigger interventions still to be defined to optimise the patient's status before undergoing TAVI.

mean gradient, left atrial diameter, red cell distribution width, comorbidities, as well as preprocedural haemoglobin and others. ${ }^{89}$ Currently, all available clinical information is being judged and weighted by an interdisciplinary heart team, consisting of an interventional cardiologist, cardiac surgeon and anaesthesiologist, to achieve the best therapy for each individual patient. In addition to this strategy, further improvement in the risk assessment for patients undergoing TAVI may be expected from biomarkers such as N-terminal probrain natriuretic peptide (NTproBNP), high-sensitivity troponin $\mathrm{T}$ (hsTNT) levels and cancer antigen (CA)125, which have been shown to provide further prognostic information. ${ }^{10-13}$ 
Table 1 Baseline characteristics of patients requiring TAVI $(n=217)$

\begin{tabular}{|c|c|c|c|}
\hline & mean $\pm S D$, median $(I Q R)$ or $n(\%)$ & & mean $\pm S D$, median (IQR) or $\%$ \\
\hline Age (years) & $82(78-86)$ & Log ES I & $20.1 \%(13.6-32.6)$ \\
\hline Male & $96(44.2 \%)$ & $\leq 20 \%$ & 107 (49.3\%) \\
\hline $\mathrm{BMI}\left(\mathrm{kg} / \mathrm{m}^{2}\right)$ & $26.2(23.7-29.1)$ & $>20 \%$ & $110(50.7 \%)$ \\
\hline NYHA classification & & ES II & $6.1 \%(3.8-10.3)$ \\
\hline I & $9(4.2 \%)$ & & \\
\hline ॥ & $61(28.1 \%)$ & & \\
\hline III & $122(56.2 \%)$ & NTproBNP (pg/mL) & $2094(932-4970.5)$ \\
\hline IV & $25(11.5 \%)$ & & \\
\hline AFIB/AFLUT & $97(44.7 \%)$ & & \\
\hline Significant CAD & $161(74.2 \%)$ & & \\
\hline Previous cardiac surgery & $87(40.1 \%)$ & & \\
\hline COPD & $39(18.0 \%)$ & & \\
\hline Diabetes & $64(29.5 \%)$ & Osteopontin (ng/mL) & $675(488.5-990.5)$ \\
\hline Dyslipidaemia & $108(49.8 \%)$ & & \\
\hline Hypertension & $197(90.8 \%)$ & & \\
\hline Peripheral vascular disease & $86(25.4 \%)$ & EF & \\
\hline Cerebrovascular disease & $33(15.2 \%)$ & $<35 \%$ & $25(11.5 \%)$ \\
\hline GFR & & $35 \%-45 \%$ & $42(19.4 \%)$ \\
\hline$<30 \mathrm{~mL} / \mathrm{min}$ & $11(5.1 \%)$ & $46 \%-54 \%$ & $41(18.9 \%)$ \\
\hline $30-45 \mathrm{~mL} / \mathrm{min}$ & $49(22.6 \%)$ & $\geq 55 \%$ & $(50.2 \%)$ \\
\hline $45-60 \mathrm{~mL} / \mathrm{min}$ & $106(48.9 \%)$ & $\begin{array}{l}\text { Systolic pulmonary } \\
\text { hypertension }\end{array}$ & $42(34-53)$ \\
\hline$>60 \mathrm{~mL} / \mathrm{min}$ & $51(23.5 \%)$ & $<35 \mathrm{~mm} \mathrm{Hg}$ & $(29.5 \%)$ \\
\hline STS PROM score & $5.2 \%(3.5-7.8)$ & $35-59 \mathrm{~mm} \mathrm{Hg}$ & $(58.5 \%)$ \\
\hline$\leq 10 \%$ & $190(87.6 \%)$ & $>59 \mathrm{~mm} \mathrm{Hg}$ & $(17.1 \%)$ \\
\hline \multirow[t]{2}{*}{$>10 \%$} & $27(12.4 \%)$ & Aortic valve area $\left(\mathrm{cm}^{2}\right)$ & $0.7(0.6-0.8)$ \\
\hline & & $A R \geq 2$, invasive & $(24.9 \%)$ \\
\hline
\end{tabular}

AFIB, atrial fibrillation; AFLUT, atrial flutter; AR, aortic regurgitation; BMI, body mass index; CAD, coronary artery disease; COPD, chronic obstructive pulmonary disease; EF, ejection fraction; ES II, EuroSCORE II; GFR, glomerular filtration rate; Log ES, logistic EuroSCORE; NTproBNP, N-terminal probrain natriuretic peptide; NYHA, New York Heart Association; STS, Society of Thoracic Surgeons; TAVI, transcatheter aortic valve implantation.

Osteopontin (OPN) is a glycoprotein that is expressed in various cell types, including cardiomyocytes and fibroblasts and can exist as an immobilised extracellular matrix molecule as well as a soluble cytokine. Because of its localisation in the myocardium and its molecular properties, OPN has been suspected to be involved in the communication between the extracellular matrix and cardiomyocytes. ${ }^{14}$ In addition, OPN is required for myofibroblast differentiation in cardiac fibrosis. ${ }^{15}$ Along these lines, OPN is markedly upregulated in several animal models of cardiac hypertrophy and heart failure ${ }^{1617}$ as well as in patients with chronic heart failure due to reduced left ventricular function, ${ }^{18}$ which implies a role in myocardial remodelling in response to biomechanical stress.

In the context of this background, we hypothesised that OPN might be of prognostic value in patients with severe symptomatic AS and cardiac hypertrophy undergoing transcatheter aortic valve replacement.

\section{METHODS}

\section{Study design and patient selection}

For the purpose of this research, we enrolled a total of 217 patients undergoing TAVI at the University Hospital Schleswig-Holstein (UKSH) Medical Centre in Kiel (Germany) between February 2011 and December 2013. ${ }^{19}$ The study was approved by the local ethics committee and conducted in accordance with the Declaration of Helsinki. All patients and healthy individuals provided written informed consent.

Eligibility for TAVI was determined based on the presence of severe symptomatic AS (as defined by mean gradient $>40 \mathrm{~mm} \mathrm{Hg}$, Aortic Valve Area (AVA) $<1.0 \mathrm{~cm}^{2}$ and/or an indexed AVA of $<0.6 \mathrm{~cm}^{2} / \mathrm{m}^{2}$, respectively), a high Log ES, EuroSCORE II (ES II) and STS PROM-Score, an age typically of more than 75 years, the presence of porcelain aorta, previous cardiac surgery or chest radiation 
A

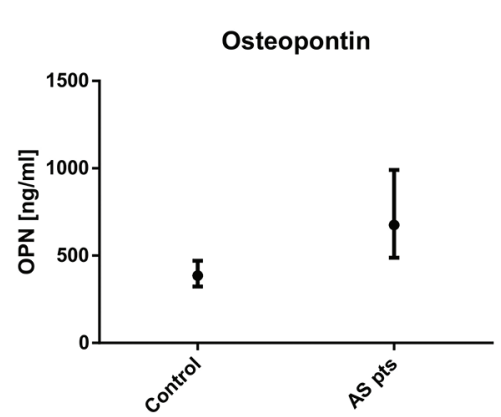

B

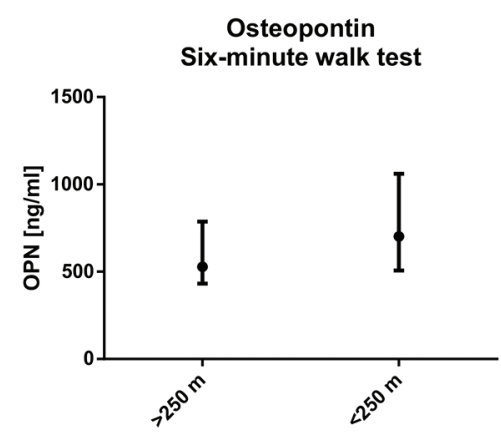

C

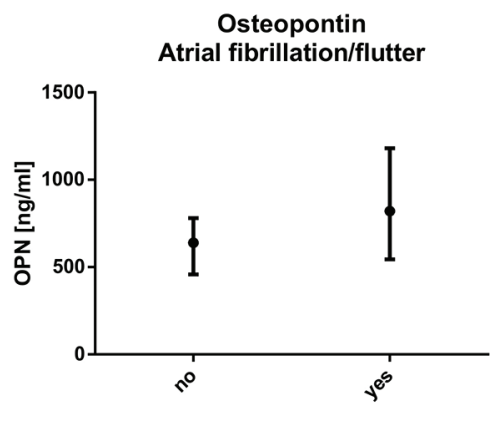

Figure 1 OPN is elevated in patients with symptomatic AS. (A) Median OPN plasma levels in patients with AS $(n=217)$ are significantly higher compared with the control sample $(\mathrm{n}=20) .386 \mathrm{ng} / \mathrm{mL}$ (IQR 323-470 ng/mL) vs $675 \mathrm{ng} / \mathrm{mL}$ (IQR 488-990.5 ng/ $\mathrm{mL}), \mathrm{p}<0.001$. (B) In patients with reduced 6 min walk test OPN levels $(702 \mathrm{ng} / \mathrm{mL}$; IQR $507-1061 \mathrm{ng} / \mathrm{mL}) \mathrm{are}$ elevated in contrast to patients with a walking distance of more than $250 \mathrm{~m}(528 \mathrm{ng} / \mathrm{mL}$; IQR $432-787 \mathrm{ng} / \mathrm{mL}), p=0.003$. (C) Levels of OPN in patients with atrial fibrillation or flutter are significantly higher $(821 \mathrm{ng} / \mathrm{mL}$; IQR $544-1181 \mathrm{ng} / \mathrm{mL})$ than in patients with sinus rhythm (639 ng/mL; IQR 458-781 ng/mL), p<0.001. AS, aortic stenosis; OPN, osteopontin.

and other comorbidities. ${ }^{20}$ The decision to perform TAVI was exclusively based on the decision of the institutional heart team. For the control group, 20 healthy individuals of a comparable age spectrum, free of any cardiac abnormalities were chosen from an institutional biobanking database. The absence of structural cardiac diseases was confirmed by echocardiography and ECG.

All patients undergoing TAVI were treated with balloon-expandable Edwards SAPIEN XT transcatheter heart valves that can be used via transfemoral (TF), transapical (TA) and transaortic (TAo) delivery. The SAPIEN $\mathrm{XT}$ is a bovine pericardial tissue valve available in 23, 26 and $29 \mathrm{~mm}$ sizes.

\section{Clinical assessment and data collection}

Before TAVI all patients underwent coronary angiography, left and right heart catheterisation. Transthoracic and transoesophageal echocardiographies were obtained before and (transthoracic only) 7 days after TAVI. In case of a significant coronary stenosis, percutaneous coronary intervention (PCI) was performed typically at least 7 days before valve implantation to prevent revascularisation-related influence on biomarker levels. Basic clinical assessment was completed by a 12-lead ECG registration, cerebrovascular duplex ultrasound, pulmonary function test and a complete laboratory work-up. In addition, all patients underwent cardiac CT scan as well as a CT angiography.

During the preprocedural clinical assessment the following supplementary parameters were collected: age, sex, body mass index (BMI), New York Heart Association (NYHA) functional classification, degree of coronary artery disease $(\mathrm{CAD})$, previous heart surgery, degree of pulmonary hypertension, comorbidities (ie, chronic obstructive pulmonary disease (COPD), diabetes, dyslipidaemia, hypertension, peripheral vascular disease, cerebrovascular disease and chronic kidney disease based on glomerular filtration rate (GFR)) and risk assessment scores (Log ES and STS PROM).
Follow-up assessments: For regular follow-up, patients were invited to yearly follow-up visits. Before data extraction, vital status or exact date of the death of the patients was confirmed by telephone or by their general practitioners.

\section{Biomarker measurement}

Blood samples were collected 1 day before TAVI and processed immediately at the in-house laboratory at UKSH Kiel. Plasma samples were stored at $-80^{\circ} \mathrm{C}$ until they were sent to our institution's core facility for biomarker measurement. OPN was measured using the Quantikine Human OPN immunoassay from R\&D Systems (Minneapolis, USA) that employs the quantitative sandwich enzyme immunoassay technique. The minimal detection dose of OPN in this assay is $0.011 \mathrm{ng} / \mathrm{mL}$. NTproBNP was measured using a system from Roche Diagnostics (Mannheim, Germany).

\section{Statistical analysis}

Dichotomous and categorical data are presented using percentages and continuous variables are summarised using standard statistics, that is, as mean and SD (for approximately normally distributed variables), or median, lower and upper quartiles (for not normally distributed variables). Associations between categorised OPN levels and other clinical variables were assessed by $\chi^{2}$ tests, t-tests or Wilcoxon rank-sum tests, as appropriate. For survival analyses, all continuous variables were dichotomised since in our experience there is often a non-linear relationship between continuous variables and survival which can be modelled by using appropriate thresholds. For clinical variables, thresholds are based on previous publications if available (such as Log ES $>20 \%$, STS $>10 \%$, GFR, ejection fraction, valve diseases) or based on upper quartile (age, BMI, ES II), since most of the variables showed skewed distributions. Therefore, measured biomarker levels (OPN, NTproBNP) were categorised in category 
$0=$ first to third quartile and category 1 =fourth quartile. All preprocedural prognostic factors, which were significant in the log-rank test, were included into a multiple Cox regression model. Following backward selection was based upon the likelihood ratio criteria. The proportional hazard assumption was checked using weighted residuals and none of the prognostic factors were found to be significant. In order to investigate whether baseline OPN values are relevant only for short-term survival (up to 6 months post TAVI) or also for longer term survival, we performed additional survival analysis based on the subsample of patients surviving at least 6 months (using only baseline variables as predictors).

All statistical analyses were performed using SPSS V.22 and the statistical software R V.3.2.2 (package survival). p Values $\leq 0.05$ were considered to be statistically significant, without adjusting for multiple comparisons.

\section{RESULTS}

\section{Baseline patient characteristics}

During the recruiting period, a total of $n=295$ patients were treated by TAVI at our centre. A total of 217 patients gave informed consent and completed the follow-up (median 349 days (IQR: 106-656 days, min 0, max 1081 days), table 1). The median age of our sample was 82 years (IQR 78-86), and 96 patients (44.2\%) were male. For the majority of patients, the TF access was chosen (56.7\%), followed by TA $(33.2 \%)$ and TAo $(10.1 \%)$.

With regard to heart failure, enrolled patients were highly symptomatic with most of the subjects falling within NYHA class III. Approximately three out of four displayed significant CAD. Moreover, about $45 \%$ of the patients had a history of atrial fibrillation/atrial flutter (AFIB/AFLUT). There were no significant differences in patient characteristics when considering the various TAVI delivery routes. The most common comorbidity was hypertension, followed by dyslipidaemia and diabetes. Many subjects had reduced kidney function (reduced calculated GFR). Mean values for C-reactive protein, creatinine and haemoglobin were within normal ranges (data not shown). The baseline median STS PROM score was 5.24\% (IQR 3.5-7.8), whereas median Log ES and median ES II were 20.1\% (IQR $13.6 \%-32.6 \%$ ) and $6.1 \%$ (IQR 3.8\%-10.3\%), respectively. Median pre-TAVI NTproBNP level was 2094 (IQR 932-4970.5) pg/mL. Approximately 1 out of 10 patients displayed severely reduced left ventricular function and a large proportion of subjects presented with significant pulmonary hypertension. Mean aortic valve area was $0.67 \mathrm{~cm}^{2}$.

A control group of 20 healthy individuals (10 male) with a median age of 79 years (IQR 77.3-81.8) was also available. The health status was proofed by the absence of relevant cardiovascular diseases or relevant cardiovascular risk factors such as diabetes. Structural heart disease was ruled out by transthoracic echocardiography.

\section{OPN in patients with severe AS and its association with clinical variables}

The median OPN plasma level in the control sample $(\mathrm{n}=20)$ was $386 \mathrm{ng} / \mathrm{mL}$ (IQR 323-470 ng/mL). Patients with AS displayed a significantly higher median OPN plasma level of $675 \mathrm{ng} / \mathrm{mL}$ (IQR 488.5-990.5 ng/mL, $\mathrm{p}<0.001$, figure $1 \mathrm{~A})$.

To assess the clinical picture associated with OPN elevations, we correlated the result of a $6 \mathrm{~min}$ walk test to OPN levels. We found a significantly higher level of OPN (702 ng/mL; IQR 507-1061) in patients with reduced 6 min walk test (lower than $250 \mathrm{~m}$ ) as compared with patients with a walking distance of more than 250 $\mathrm{m}$ (OPN levels of $528 \mathrm{ng} / \mathrm{mL}$; IQR 432-787, $\mathrm{p}=0.003$, figure $1 \mathrm{~B})$. The absolute distance of the $6 \mathrm{~min}$ walk test correlated negatively with absolute OPN levels $(\mathrm{r}=-0.24$; $\mathrm{p}<0.001)$. The classification of the level of heart failure based on the NYHA functional class showed no significant relation to OPN levels $(\mathrm{p}=0.588)$.

Finally, we found significantly elevated levels of OPN in patients with AFIB or AFLUT $(821 \mathrm{ng} / \mathrm{mL}$; IQR 544-1181) in contrast to patients with sinus rhythm (639 ng/mL; IQR 458-781; $\mathrm{p}<0.001$, figure 1C).

\section{Variables associated with elevated preoperative OPN levels}

To further understand which variables might influence or might be associated with elevated pre-TAVI OPN levels, we compared patient groups with lower versus higher OPN levels (first to third quartile vs fourth quartile) (table 2). The well-known prognostic factors AFIB/ AFLUT $(\mathrm{p}=0.003)$, elevated STS PROM score $(>10 \%$, $\mathrm{p}=0.007)$, Log ES $(>20 \%, \mathrm{p}=0.008)$, ES II (upper quartile, $\mathrm{p}=0.044)$, preprocedural aortic valve gradient mean $(\mathrm{p}=0.035)$ and several Valve Academic Research Consortium-2 (VARC-2) criteria were linked to higher OPN levels. No procedural variables were associated with elevated OPN levels. All recorded VARC-2 criteria, stratified into the two groups with lower versus higher OPN levels (first to third quartile vs fourth quartile), are shown in table 3.

\section{Analysis of predictors of survival in patients undergoing aortic valve replacement by TAVI}

In the next step, we analysed which factors were associated with mortality in this patient cohort. In table 4 we summarised the results of the comparison of survival times for all variables individually. Significantly increased risk for premature death was observed in patients presenting with AFIB or AFLUT $(\mathrm{p}=0.018)$ and COPD $(\mathrm{p}=0.009)$. Moreover, we found that an elevated STS PROM score $>10 \%(p=0.017), \log$ ES $>20 \%$ $(\mathrm{p}<0.001)$, upper quartile of ES II $(>10.3 \%, \mathrm{p}=0.016)$, preprocedural NTproBNP levels in the upper quartile $(\mathrm{p}=0.035)$, TAo and TA access (compared with TF) and some VARC-2 outcomes (including kidney injury) were associated with increased mortality after the TAVI procedure. 
Table 2 Association of elevated pre-TAVI OPN levels with clinical variables

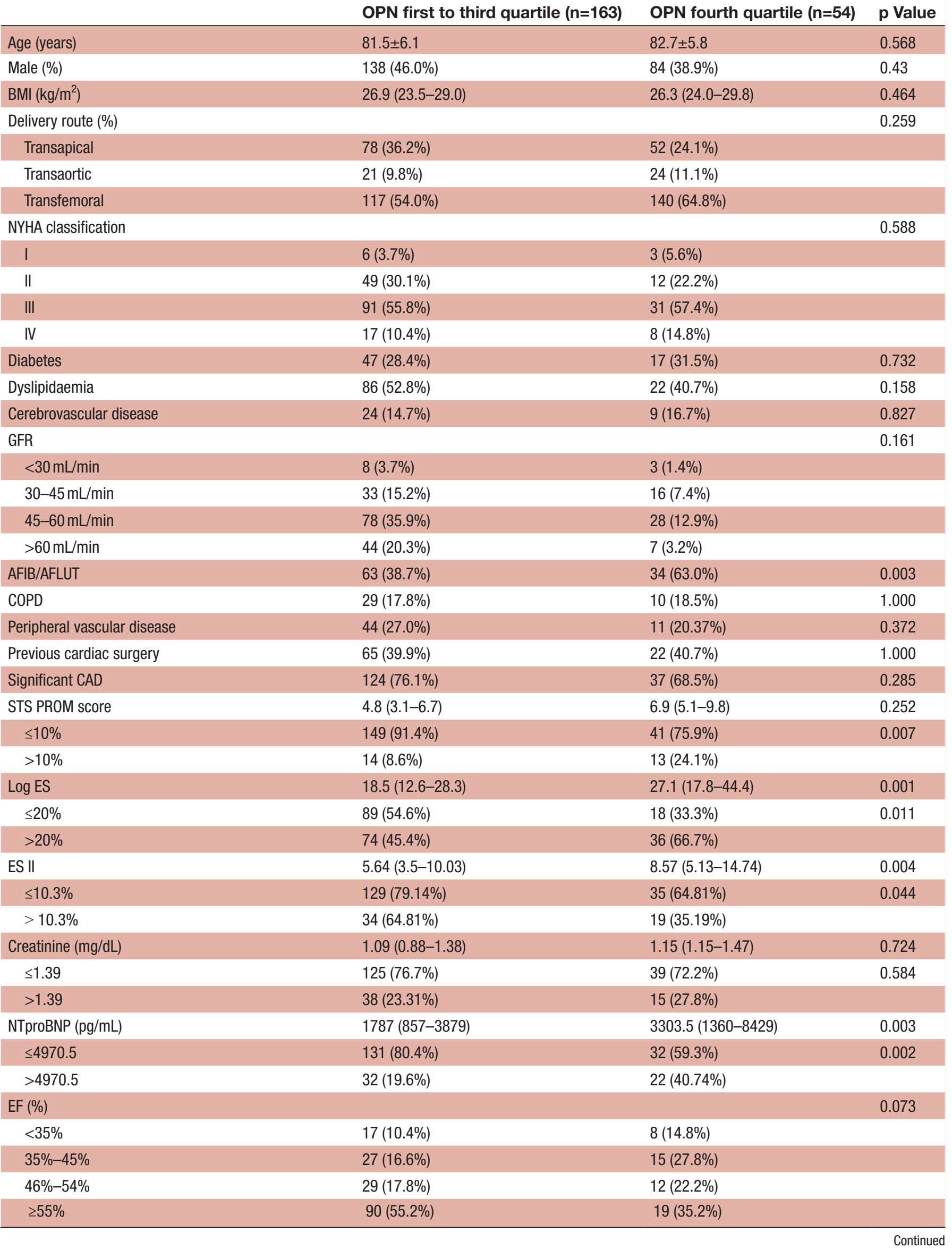




\section{Table 2 Continued}

\begin{tabular}{|c|c|c|c|}
\hline & OPN first to third quartile $(n=163)$ & OPN fourth quartile $(n=54)$ & p Value \\
\hline 30-day mortality & $9(5.52 \%)$ & $6(11.11 \%)$ & 0.212 \\
\hline
\end{tabular}

AFIB, atrial fibrillation; AFLUT, atrial flutter; AVG, aortic valve gradient; BMI, body mass index; CAD, coronary artery disease; COPD, chronic obstructive pulmonary disease; EF, ejection fraction; ES II, EuroSCORE II; GFR, glomerular filtration rate; Log ES, logistic EuroSCORE; NTproBNP, N-terminal probrain natriuretic peptide; NYHA, New York Heart Association; OPN, osteopontin; STS, Society of Thoracic Surgeons; TAVI, transcatheter aortic valve implantation.

Importantly, OPN levels in the highest quartile also showed a significant association with reduced survival time after TAVI $(p=0.002)$. Figure 2 illustrates the Kaplan-Meier curves based on dichotomised OPN levels. Panel A shows the upper quartile of OPN compared with the lower three quartiles (prespecified cut-off). Even when stratifying OPN levels into all four quartiles the uppermost (fourth) quartile demonstrated a significant difference compared with the other quartiles emphasising the selectivity of this cut-off (figure 2B), $\mathrm{p}=0.019$ (log rank test). Interestingly, preoperative OPN levels are however only prognostic for survival within the first 6 months after TAVI. Within the subgroup of patients still alive after 6 months, baseline OPN levels are not related to further survival $(\mathrm{p}=0.32, \log$ rank test, figure 2C).

\section{OPN for risk stratification of postprocedural survival}

For the evaluation of OPN as a predictor of reduced survival in combination with other (well-known) mortality-associated factors, a Cox regression analysis was performed (table 5). Similar to the single-factor Kaplan-Meier analyses combined with log rank tests, Cox analysis was used to compare the upper quartile to the lower three quartiles of continuous variables. This analysis shows that increased OPN levels considered individually have a HR for adverse outcome of 2.15 (95\% CI 1.3 to $3.5, \mathrm{p}=0.002)$. In subsequently performed multiple Cox regression analysis, surprisingly NTproBNP did not remain significant when adjusted for OPN. In contrast, the upper quartile of OPN itself revealed an HR of 2.06 (CI 1.2 to 3.5, $\mathrm{p}=0.005)$. Moreover, our analysis revealed that Log ES

Table 3 Postprocedural outcome according to the VARC-2 criteria

\begin{tabular}{lccc}
\hline & OPN first to third quartile $(\mathbf{n}=\mathbf{1 6 3})$ & OPN fourth quartile $(\mathbf{n}=\mathbf{5 4})$ & $\mathbf{p}$ Value \\
\hline 30-day mortality & $9(5.52 \%)$ & $6(11.11 \%)$ & 0.212 \\
\hline VARC-2 myocardial infarction & $3(1.8 \%)$ & $1(1.9 \%)$ & 0.996 \\
\hline VARC-2 stroke & $8(4.5 \%)$ & $1(1.9 \%)$ & 0.457 \\
\hline VARC-2 disabling stroke & $5(3.1 \%)$ & 0 & 0.335 \\
\hline VARC-2 bleeding & $23(14.1 \%)$ & $13(24.1 \%)$ & 0.095 \\
\hline VARC-2 life-threatening bleeding & $6(3.7 \%)$ & $2(3.7 \%)$ & 1.000 \\
\hline VARC-2 major bleeding & $10(6.1 \%)$ & $10(18.5 \%)$ & 0.012 \\
\hline VARC-2 acute kidney injury & $4(2.5 \%)$ & $7(13.0 \%)$ & 0.006 \\
\hline VARC-2 acute kidney injury stage 3 & $1(0.6 \%)$ & $5(9.3 \%)$ & 0.004 \\
\hline VARC-2 vascular complications & $22(13.5 \%)$ & $9(16.7 \%)$ & 0.654 \\
\hline VARC-2 major vascular complications & $3(1.8 \%)$ & $1(1.9 \%)$ & 1.000 \\
\hline VARC-2 persistent high-degree AV block & $9(5.5 \%)$ & $3(5.6 \%)$ & 1.000 \\
\hline VARC-2 pacemaker implantation & $11(6.7 \%)$ & $5(9.3 \%)$ & 0.306 \\
\hline VARC-2 conversion to open surgery & $1(0.6 \%)$ & $1(1.9 \%)$ & 0.437 \\
\hline VARC-2 TAVI-in-TAVI deployment & $5(3.1 \%)$ & $4(7.4 \%)$ & 0.231 \\
\hline Valve regurgitation post TAVI & & & 0.204 \\
\hline 0 & $61(37.42 \%)$ & $18(33.33 \%)$ & $3(55.56 \%)$ \\
\hline I & $78(47.85 \%)$ & $5(9.26 \%)$ & $1(1.85 \%)$ \\
\hline II & $24(14.72 \%)$ & 0 & \\
\hline III & 0 & & \\
\hline AV & & & \\
\hline
\end{tabular}

AV, atrioventricular; OPN, osteopontin; TAVI, transcatheter aortic valve implantation; VARC-2, Valve Academic Research Consortium-2. 
Table 4 Association of baseline variables with mortality until last follow-up

\begin{tabular}{|c|c|}
\hline & p Value (log-rank test) \\
\hline Age & 0.950 \\
\hline Male & 0.548 \\
\hline BMI & 0.182 \\
\hline NYHA classification & 0.483 \\
\hline Delivery route & 0.048 \\
\hline AFIB/AFLUT & 0.018 \\
\hline Significant CAD & 0.098 \\
\hline Previous cardiac surgery & 0.820 \\
\hline COPD & 0.009 \\
\hline Diabetes mellitus & 0.182 \\
\hline Dyslipidaemia & 0.428 \\
\hline Hypertension & 0.961 \\
\hline Peripheral vascular disease & 0.682 \\
\hline Cerebrovascular disease & 0.063 \\
\hline GFR & 0.172 \\
\hline STS PROM score $>10 \%$ & 0.017 \\
\hline $\log E S>20 \%$ & $<0.001$ \\
\hline ES II >10.3\% (Q4) & 0.016 \\
\hline NTproBNP > 4970.5 pg/mL (Q4) & 0.035 \\
\hline OPN >990.5ng/mL (Q4) & 0.002 \\
\hline EF (category) & 0.778 \\
\hline Systolic pulmonary hypertension & 0.238 \\
\hline Aortic valve area & 0.387 \\
\hline Preprocedural AR invasive $\geq 2$ & 0.410 \\
\hline Post-TAVI AR $\geq 2$ & 0.153 \\
\hline Acute kidney injury (VARC-2) & $<0.001$ \\
\hline Acute kidney injury, stage 3 & $<0.001$ \\
\hline
\end{tabular}

AFIB, atrial fibrillation; AFLUT, atrial flutter; AR, aortic regurgitation; $\mathrm{BMI}$, body mass index; CAD, coronary artery disease; COPD, chronic obstructive pulmonary disease; EF, ejection fraction; ES II, EuroSCORE II; GFR, glomerular filtration rate; Log ES, logistic EuroSCORE; NTproBNP, N-terminal probrain natriuretic peptide; NYHA, New York Heart Association; OPN, osteopontin; STS, Society of Thoracic Surgeons; TAVI, transcatheter aortic valve implantation; VARC, Valve Academic Research Consortium-2.

(HR 2.09, CI 1.2 to 3.5; $\mathrm{p}=0.005$ ), AFIB/AFLUT (HR 1.71 , CI 1.1 to $3.0 ; \mathrm{p}=0.046$ ), TA access (when compared with $\mathrm{TF}$ access) and COPD were the other independent risk factors for reduced survival. Among patients who survived at least 6 months after TAVI, the only baseline risk factor that was associated with further survival was COPD $(p=0.007)$.

\section{Effect of combination of OPN with NTproBNP}

We analysed the effects of the combination of the two humeral biomarkers NTproBNP and OPN. More often than expected by chance, these biomarkers were concordant in patients undergoing TAVI (both elevated or both not elevated, $\mathrm{p}=0.002$ for association of NTproBNP and OPN, see table 2). Elevation of both factors (high BNP and high OPN) leads to a strong, significant increase of the HR to 2.82 (95\% CI 1.5 to $5.4, \mathrm{p}=0.002$ ) indicating joint effects of OPN and NTproBNP (see table 6). Risks associated with just one elevated biomarker were lower and not significant with the small number of discordant patients available. Estimated HRs are compatible with a multiplicative risk model. The corresponding Kaplan-Meier survival curves are shown in figure 3.

\section{DISCUSSION}

Aortic valve stenosis is the most prevalent form of heart valve disease leading to surgical valve replacement in the western world..$^{21}$ One of the principal clinical problems and indicators of an increased risk is the development and progression of left ventricular hypertrophy as well as the progress towards chronic heart failure. As described above, it has been shown before that OPN levels are markedly upregulated in cardiac hypertrophy, heart failure and amyloidosis, and therefore associated with decompensation and death. ${ }^{18}$ ${ }^{23}$ In this study, we measured plasma OPN levels in patients with severe symptomatic AS prior to TAVI. Given its upregulation in left ventricular hypertrophy and fibrosis, ${ }^{23}$ OPN levels might help identify patients at highest risk for decompensation or death. The aim was to evaluate the potential of OPN as a biomarker for predicting mortality. The presented findings might help improve preprocedural risk stratification and detection of patients at high risk for adverse outcome prior to aortic valve replacement.

\section{Role of OPN in cardiac fibrosis and hypertrophy}

The results shown here for patients with severe symptomatic AS are concordant with data from our group wherein increased OPN levels have been described in adult patients with heart failure. ${ }^{19}$ Rosenberg et al illustrated that elevated OPN levels were associated with a higher 4-year death rate in patients with heart failure. ${ }^{18}$ In an additional analysis of NTproBNP levels together with OPN levels, Rosenberg presented a significantly increased 4-year death rate from $50 \%$ (elevated NTproBNP only) to $73 \%$ (elevated NTproBNP and elevated OPN).

Our findings are also consistent with elevated OPN levels in several animal models of cardiac disease. We were able to show earlier that mice with left ventricular hypertrophy caused by overexpression of constitutively active calcineurin A or chronic angiotensin II infusion exhibited significant upregulation of cardiac OPN. ${ }^{18}$ Further, Matsui et al showed that OPN expression was significantly enhanced in mice with inducted cardiac hypertrophy due to increased mechanical stress. ${ }^{24}$ Pathophysiologically, OPN levels are upregulated by angiotensin II, which itself represents an important inductor of cardiac fibrosis. ${ }^{23}$ Conversely, experimental studies with OPN knockout mice revealed that 
A

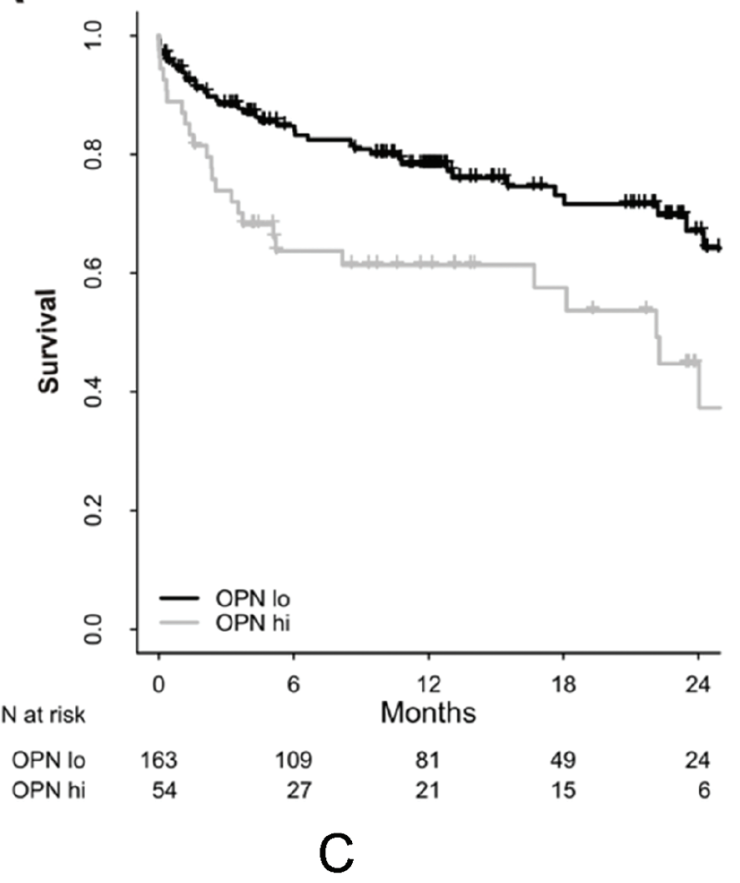

B

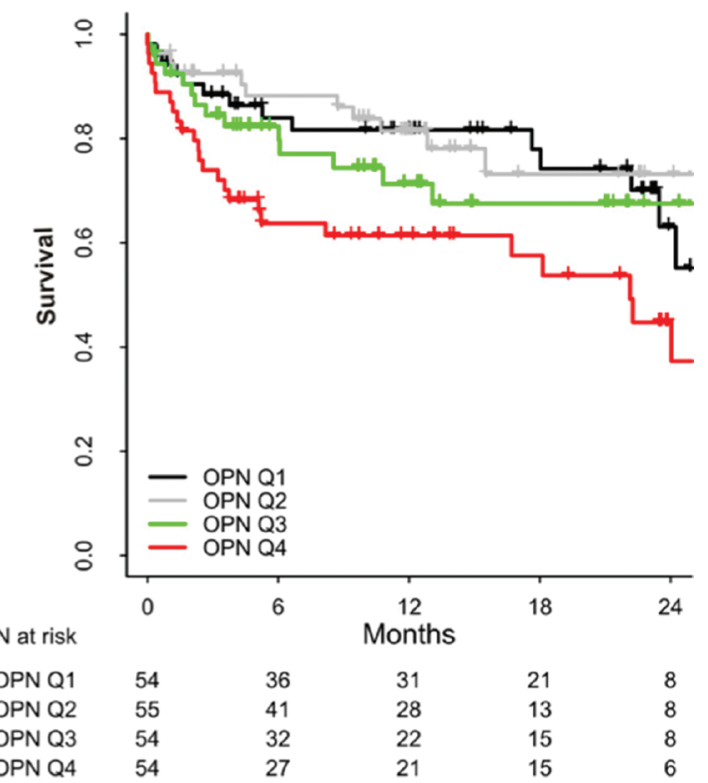

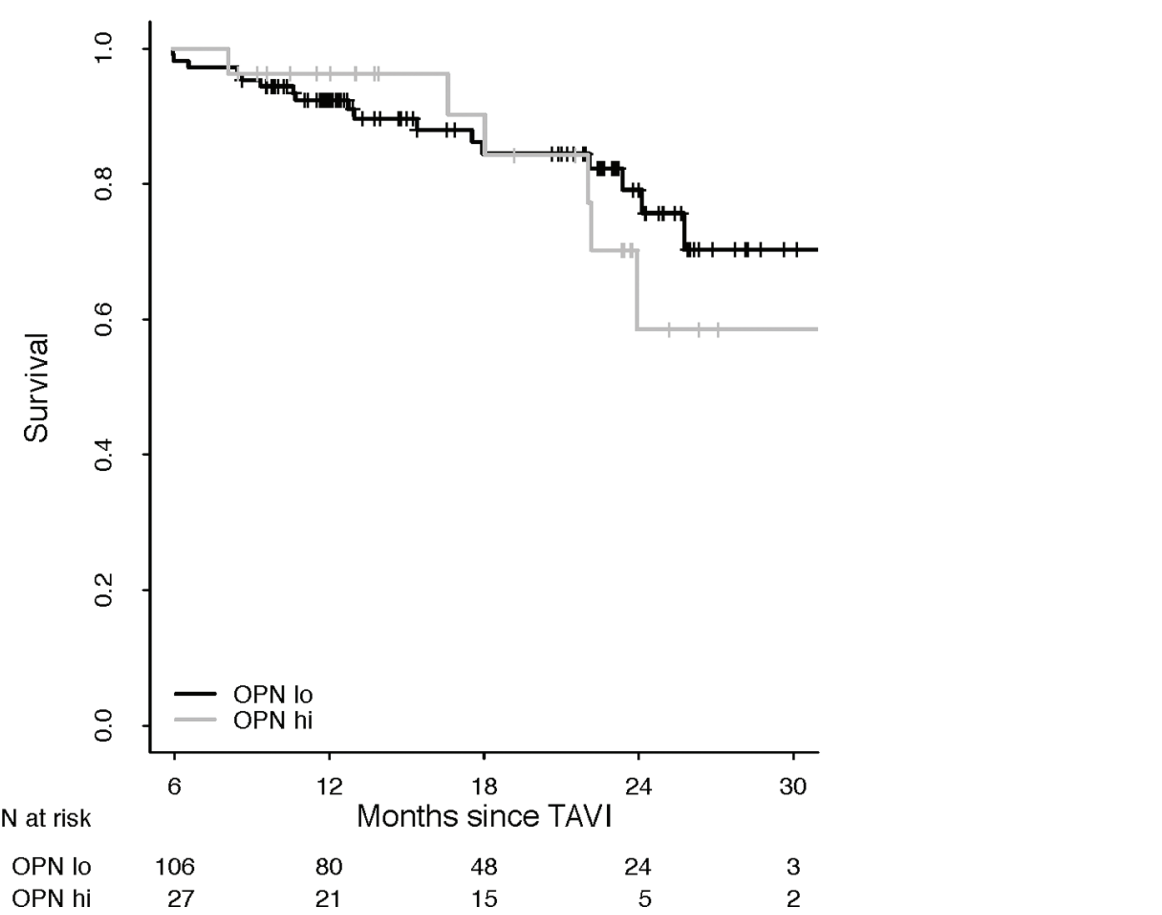

Figure 2 Kaplan-Meier curves based on OPN levels. (A) Upper quartile of OPN compared with the lower three quartiles. (B) Stratification of OPN levels into all four quartiles. (C) Kaplan-Meier curve for OPN upper quartile compared with the lower three quartiles, beginning at 6 months post TAVI. OPN, osteopontin; TAVI, transcatheter aortic valve implantation.

OPN deficiency abolished angiotensin II-induced cardiac fibrosis. ${ }^{25}$ OPN also plays a critical role in the Ang-II-dependent upregulation of miR-21 in cardiac fibrosis. ${ }^{26}$

\section{Role of OPN in TAVI risk stratification}

The use of biomarkers in preoperative or preprocedural risk stratification in patients with AS is still being discussed. Besides evaluation of short-term effects such as intraprocedural assessment of aortic regurgitation, ${ }^{27}$ biomarkers are typically considered as markers of longterm prognosis. Thus, implementation of biomarkers in the risk stratification together with the typical clinical scores such as the Log ES, ES II or the STS score might add helpful information for the optimal choice of the method used (conventional vs TAVI). In this context, biomarkers such as hsTNT, NTproBNP, CA-125 and 
Table 5 Results of survival time analyses in patients undergoing TAVI (multiple Cox regression analysis)

\begin{tabular}{|c|c|c|c|c|}
\hline Tested variables & Crude HR (95\% Cl) & p Value & Adjusted HR $(95 \% \mathrm{Cl})$ & p Value (Cox) \\
\hline NTproBNP >4970.5 & 1.71 (1.0 to 2.8) & 0.037 & & ns \\
\hline OPN > 990.5 & 2.15 (1.31 to 3.53$)$ & 0.002 & 2.06 (1.2 to 3.5$)$ & 0.005 \\
\hline Log ES > 20\% & 2.41 (1.5 to 4.0$)$ & 0.001 & 2.09 (1.24 to 3.51$)$ & 0.005 \\
\hline ES $\|>10.3 \%$ & 1.89 (1.1 to 3.2$)$ & 0.018 & & ns \\
\hline STS PROM score $>20 \%$ & 2.03 (1.1 to 3.7$)$ & 0.019 & & ns \\
\hline AFIB/AFLUT & 1.80 (1.1 to 3.0$)$ & 0.020 & 1.71 (1.04 to 2.83$)$ & 0.046 \\
\hline COPD & 1.99 (1.2 to 3.4$)$ & 0.011 & 1.77 (1.04 to 3.04$)$ & 0.036 \\
\hline TA access ${ }^{*}$ & 1.59 (1.0 to 2.7$)$ & 0.075 & 2.01 (1.12 to 3.42$)$ & 0.011 \\
\hline TAo access* & $2.43(1.1$ to 5.6$)$ & 0.038 & 2.19 (0.94 to 5.11$)$ & 0.071 \\
\hline
\end{tabular}

${ }^{*}$ Compared with transfemoral.

AFIB, atrial fibrillation; AFLUT, atrial flutter; COPD, chronic obstructive pulmonary disease; ES II, EuroSCORE II; Log ES, logistic EuroSCORE; ns, non-significant; NTproBNP, N-terminal probrain natriuretic peptide; OPN, osteopontin; STS, Society of Thoracic Surgeons; TA, transapical; Tao, transaortic; TAVI, transcatheter aortic valve implantation.

others have already been shown to have relevant value in risk stratification ${ }^{11-13} 19$ and may be further supported by OPN. Surprisingly, the OPN levels did not correlate significantly with the extent of heart failure, as measured by NYHA classification.

The findings of the present study revealed that in patients suffering from severe symptomatic aortic valve stenosis and undergoing TAVI increased OPN levels are associated with higher mortality and premature death within the first 6 months. More importantly, OPN levels provided prognostic information independently from clinical risk scores and other biomarkers. Nevertheless, the combination of OPN with NTproBNP revealed a joint effect of OPN with both biomarkers leading to additional prognostic value.

In summary, OPN represents a useful and powerful biomarker providing additional prognostic information in addition to established clinical parameters in patients with severe aortic valve stenosis and TAVI procedure. Further 'multibiomarker' analyses are needed to identify an optimal combination of biomarkers and to further evaluate their potential role in risk stratification. So far,

Table 6 Analysis of joint effects of pre-TAVI NTproBNP and pre-TAVI OPN levels

\begin{tabular}{|c|c|c|c|}
\hline Group & $\mathbf{n}$ & HR $(95 \% \mathrm{Cl})$ & p Value \\
\hline (1) NTproBNP low, OPN low & 131 & 1 & \\
\hline $\begin{array}{l}\text { (2) NTproBNP high, OPN } \\
\text { low }\end{array}$ & 32 & 1.41 (0.69 to 2.89$)$ & ns \\
\hline $\begin{array}{l}\text { (3) NTproBNP low, OPN } \\
\text { high }\end{array}$ & 32 & 1.92 (0.86 to 3.77$)$ & ns \\
\hline $\begin{array}{l}\text { (4) NTproBNP high, OPN } \\
\text { high }\end{array}$ & 22 & 2.82 (1.48 to 5.37$)$ & 0.002 \\
\hline
\end{tabular}

ns, non-significant; NTproBNP, N-terminal probrain natriuretic peptide; OPN, osteopontin; TAVI, transcatheter aortic valve implantation. the clinical view and use of clinical scores supported by biomarkers takes an important place in preprocedural risk assessment. This could give more evidence in the decisions made by the heart teams and facilitate the process of identifying high-risk patients. These patients could be analysed and treated in an appropriated way to address their elevated periprocedural mortality risk. New biomarkers appear to support a beneficial role in risk stratification.

\section{Limitations}

Limitations of the present study are as follows: (1) Our sample size with more than 200 patients might still not be sufficient for adequate OPN risk assessment. (2) Not all consecutive patients undergoing TAVI could be recruited for the registry in this period of time (217 out of 295). (3) Our investigation was conducted as a single-centre trial. However, in a single centre many procedures are standardised as opposed to a multicentre situation where the risk of unknown bias is high. (4) By choosing to not adjust for multiple comparisons the risk of type 1 error increases. (5) The device that has been used (Edwards Lifesciences Sapien $\mathrm{XT}$ ) is largely replaced by the Edwards Lifesciences Sapien-3, but the main focus of this study was to evaluate the risk of patients with severe AS, independent of the type of the valve prosthesis used. (6) As OPN is not cardiac specific and is elevated in other conditions, there might be increased mortality as a result of comorbidity. (7) Myocardial mass as a reliable parameter of cardiac hypertrophy was not measured routinely in these patients. Thus, a precise correlation of OPN values with cardiac hypertrophy is not possible.

\section{CONCLUSIONS}

OPN levels are elevated in patients with AS and correlate with several clinical parameters. Furthermore, OPN is 


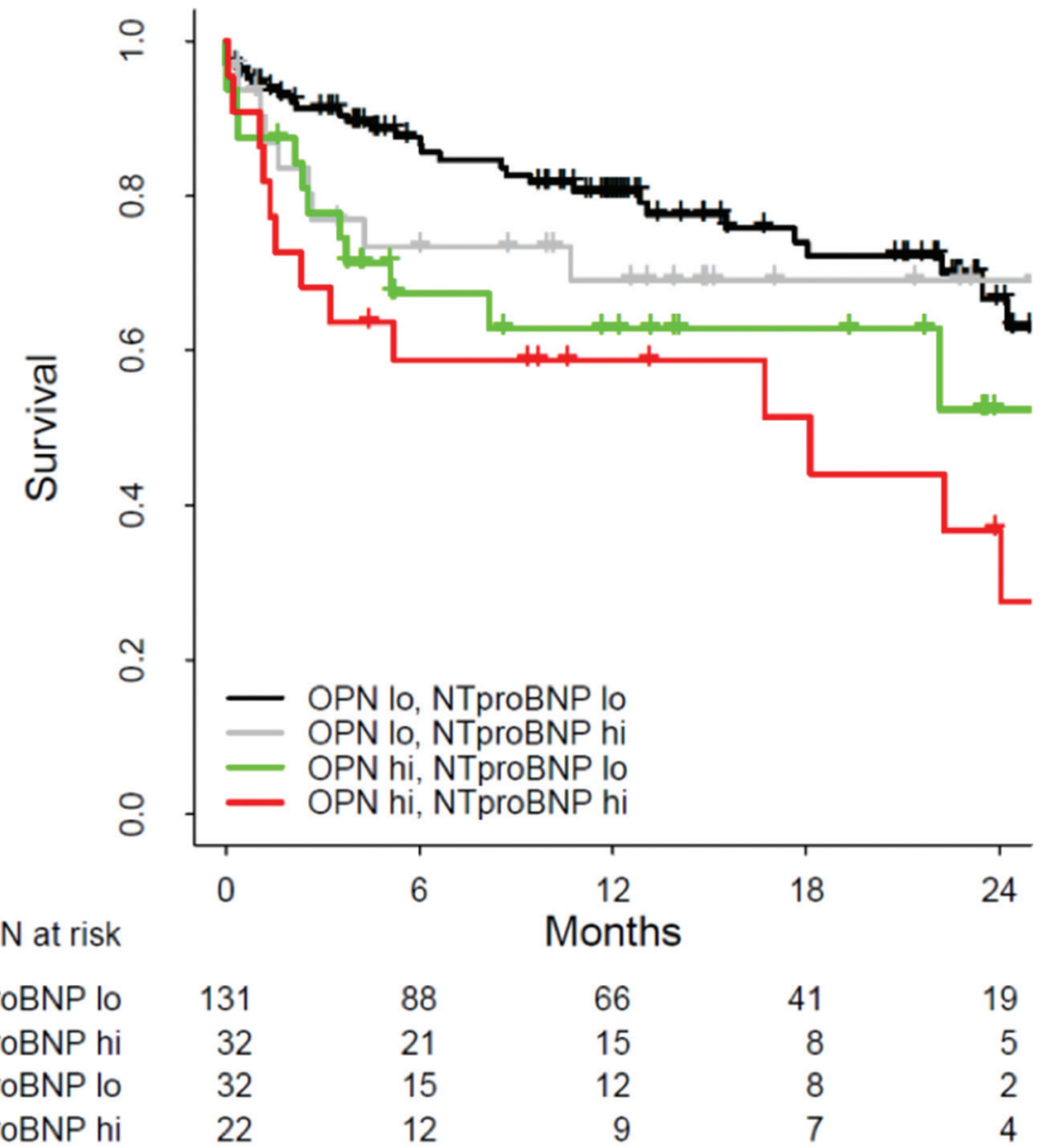

Figure 3 Analysis of joint effects of pre-TAVI NTproBNP and pre-TAVI OPN levels, Kaplan-Meier curves. NTproBNP, N-terminal probrain natriuretic peptide; OPN, osteopontin; TAVI, transcatheter aortic valve implantation.

a strong predictor of mortality in patients undergoing TAVI and can expand the prognostic power of established biomarkers in these patients. Future studies should focus on the evaluation of OPN's capability in advancing the clinical judgement of patients with AS.

Contributors Study conception and design: ML, DF. Acquisition of data: Nvl, SFW. Analysis and interpretation of data: ML, Nvl, SFW, AD, PB, DF. Drafting of manuscript: ML, MLa, JF, DF. Critical revision: ML, MR, GL, JF, PB, NF, DF.

Competing interests None declared.

Patient consent Detail has been removed from this case description/these case descriptions to ensure anonymity. The editors and reviewers have seen the detailed information available and are satisfied that the information backs up the case the authors are making.

Ethics approval The study was approved by the local ethics committee and conducted in accordance with the Declaration of Helsinki. All patients and healthy individuals provided written informed consent.

Provenance and peer review Not commissioned; internally peer reviewed.

Data sharing statement № further unpublished data are available. All data are published or under review.

Open Access This is an Open Access article distributed in accordance with the Creative Commons Attribution Non Commercial (CC BY-NC 4.0) license, which permits others to distribute, remix, adapt, build upon this work non-commercially, and license their derivative works on different terms, provided the original work is properly cited and the use is non-commercial. See: http://creativecommons.org/licenses/by-nc/4.0/

(c) Article author(s) (or their employer(s) unless otherwise stated in the text of the article) 2017. All rights reserved. No commercial use is permitted unless otherwise expressly granted.

\section{REFERENCES}

1. Litzler PY, Borz B, Smail H, et al. Transapical aortic valve implantation in Rouen: four years' experience with the Edwards transcatheter prosthesis. Arch Cardiovasc Dis 2012;105:141-5.

2. Thomas M, Schymik G, Walther T, et al. Thirty-day results of the SAPIEN Aortic Bioprosthesis European Outcome (SOURCE) Registry: a European registry of transcatheter Aortic valve implantation using the Edwards SAPIEN valve. Circulation 2010;122:62-9.

3. Wendler $\mathrm{O}$, Walther $\mathrm{T}$, Nataf $\mathrm{P}$, et al. Trans-apical aortic valve implantation: univariate and multivariate analyses of the early results from the SOURCE registry. Eur J Cardiothorac Surg 2010;38:119-27.

4. Nashef SAM, Roques F, Sharples LD, et al. EuroSCORE II. European Journal of Cardio-Thoracic Surgery 2012;41:734-45.

5. O'Brien SM, Shahian DM, Filardo G, et al. The Society of Thoracic Surgeons 2008 cardiac surgery risk models: part 2--isolated valve surgery. Ann Thorac Surg 2009;88(1 Suppl):S23-S42.

6. Piazza N, Wenaweser P, van Gameren M, et al. Relationship between the logistic EuroSCORE and the Society of Thoracic Surgeons predicted risk of mortality score in patients implanted with the CoreValve ReValving system-a Bern-Rotterdam Study. Am Heart $J$ 2010;159:323-9.

7. Roques F, Michel P, Goldstone AR, et al. The logistic EuroSCORE. Eur Heart J 2003;24:882-2.

8. Gotzmann M, Thiessen A, Lindstaedt M, et al. Left atrial diameter, aortic mean gradient, and hemoglobin for risk stratification in patients undergoing transcatheter aortic valve implantation. Clin Cardiol 2013;36:228-34.

9. Aung N, Dworakowski R, Byrne J, et al. Progressive rise in red cell distribution width is associated with poor outcome after transcatheter aortic valve implantation. Heart 2013;99:1261-6.

10. Yamamoto M, Hayashida K, Mouillet G, et al. Prognostic value of chronic kidney disease after transcatheter aortic valve implantation. J Am Coll Cardiol 2013;62:869-77. 
11. Frank D, Stark S, Lutz M, et al. Preprocedural high-sensitive troponin predicts survival after transcatheter aortic valve implantation (TAVI). Int J Cardiol 2013;169:e38-e39.

12. Köhler WM, Freitag-Wolf S, Lambers M, et al. Preprocedural but not periprocedural high-sensitive troponin $\mathrm{T}$ levels predict outcome in patients undergoing transcatheter aortic valve implantation. Cardiovasc Ther 2016;34:385-96.

13. Husser $O$, Núñez J, Núñez E, et al. Tumor marker carbohydrate antigen 125 predicts adverse outcome after transcatheter aortic valve implantation. JACC Cardiovasc Interv 2013;6:487-96.

14. Okamoto H. Osteopontin and cardiovascular system. Mol Cell Biochem 2007:300(1-2):1-7.

15. Lenga $\mathrm{Y}, \mathrm{Koh} A$, Perera AS, et al. Osteopontin expression is required for myofibroblast differentiation. Circ Res 2008;102:319-27.

16. Singh K, Sirokman G, Communal C, et al. Myocardial osteopontin expression coincides with the development of heart failure. Hypertension 1999;33:663-70.

17. Graf K, Do YS, Ashizawa N, et al. Myocardial osteopontin expression is associated with left ventricular hypertrophy. Circulation 1997;96:3063-71.

18. Rosenberg M, Zugck C, Nelles M, et al. Osteopontin, a new prognostic biomarker in patients with chronic heart failure. Circ Heart Fail 2008;1:43-9.

19. Krau NC, Lünstedt NS, Freitag-Wolf S, et al. Elevated growth differentiation factor 15 levels predict outcome in patients undergoing transcatheter aortic valve implantation. Eur J Heart Fail 2015; 17:945-55

20. Kuck KH, Hamm C. Executive summary of the position paper of the german cardiac society on quality criteria for the implementation of transcatheter aortic valve implantation (TAVI. Eur Heart $J$ 2015;36:328-30.

21. lung $B$, Baron $G$, Butchart $E G$, et al. A prospective survey of patients with valvular heart disease in Europe: the Euro Heart survey on valvular Heart Disease. Eur Heart J 2003;24:1231-43.

22. Nkomo VT, Gardin JM, Skelton TN, et al. Burden of valvular heart diseases: a population-based study. Lancet 2006;368:1005-11.

23. Frangogiannis NG. Matricellular proteins in cardiac adaptation and disease. Physiol Rev 2012;92:635-88.

24. Matsui $\mathrm{Y}$, Jia N, Okamoto $\mathrm{H}$, et al. Role of osteopontin in cardiac fibrosis and remodeling in angiotensin Il-induced cardiac hypertrophy. Hypertension 2004;43:1195-201.

25. Collins AR, Schnee J, Wang W, et al. Osteopontin modulates angiotensin II-induced fibrosis in the intact murine heart. J Am Coll Cardiol 2004;43:1698-705.

26. Lorenzen JM, Schauerte $\mathrm{C}$, Hübner $\mathrm{A}$, et al. Osteopontin is indispensible for AP1-mediated angiotensin II-related miR-21 transcription during cardiac fibrosis. Eur Heart J 2015;36:2184-96.

27. Van Belle E, Rauch A, Vincent F, et al. Von Willebrand factor multimers during transcatheter Aortic-Valve Replacement. $N$ Engl J Med 2016;375:335-44. 